\title{
Valoración de una propuesta educativa para el desarrollo del razonamiento algebraico empleando la noción de linealidad
}

\author{
Cecilia Gaita \\ Raúl Supo \\ Francisco Ugarte
}

\begin{abstract}
Resumen: Se valora la propuesta de una institución escolar, en términos de la evolución del Razonamiento Algebraico Elemental (RAE), teniendo como centro de interés la noción de linealidad. Esta valoración se hace a partir de los significados de la linealidad presentes en la formación básica (primaria y secundaria) y a la adaptación de los niveles del RAE a dicha noción. Los resultados determinan que en dicha institución la noción de linealidad

Cecilia Gaita

Doutora em Didáctica de la Matemática pela Universidade de Valladolid (UVa), España.

Professora da Pontificia Universida Católica del Perú (PUCP), Lima, Perú. se desarrolla en los diferentes grados y que, si bien se propicia su evolución, esto sólo se hace hasta el tercer nivel de algebrización. Así, el aporte fundamental consiste en articular los significados de la linealidad y del RAE para analizar una propuesta educativa particular.
\end{abstract}

http://orcid.org/0000-0002-7827-9262 $\triangle$ cgaita@pucp.edu.pe

Raúl Supo

Mestre em Enseñanza de las Matemáticas pela Pontificia Universidad Católica del Perú (Perú). Professor do Innova Schools, Lima, Perú.

iD https://orcid.org/0000-0003-2639-9569 $\bowtie$ a20194368@pucp.edu.pe

Francisco Ugarte Doutor em Matemáticas pela Universidade de Valladolid (UVa), España.Professor da Pontificia Universida Católica del Perú (PUCP), Lima, Perú

iD https://orcid.org/0000-0001-5071-8924 $\triangle$ fugarte@pucp.edu.pe

Recebido em 29/06/2021 Aceito em 09/09/2021 Publicado em 31/10/2021
Palabras clave: Enfoque Ontosemiótico. Razonamiento Algebraico. Linealidad.

\section{Assesment of an educational proposal for the development of the EAR through the notion of linearity}

Abstract: The proposal of a school institution is valued, in terms of the evolution of Elemental Algebraic Reasoning (EAR), having the notion of linearity as its center of interest. This assessment is made from the meanings of linearity present in basic education (primary and secondary) and the adaptation of the EAR levels to this notion. The results determine that in said institution the notion of linearity is developed in different degrees and that, although its evolution is encouraged, this is only done up to the third level of algebrization. Thus, the fundamental contribution consists in articulating the meanings of linearity and EAR to analyze a particular educational proposal.

Keywords: Onto-semiotic approach. Algebraic reasoning. Linearity.

\section{Avaliação de uma proposta educacional para o desenvolvimento do raciocínio algébrico a partir da noção de linearidade}

Resumo: Apresentamos os resultados da avaliação da proposta de uma instituição escolar, no que se refere à evolução do Raciocínio Algébrico Elementar (RAE), tendo como foco de atenção a noção de linearidade. A noção de configuração ontosemiótica permitiu a construção de um significado de referência da linearidade na educação básica peruana (fundamental e médio) e os níveis do RAE foram adaptados a essa noção para avaliar sua prática matemática. Os resultados determinam que nesta instituição a noção de linearidade se desenvolve nos diferentes anos do ensino básico e médio e que, embora sua seja promovida, esta só se faz no terceiro nível de algebrização. Assim, a 
contribuição fundamental consiste em articular os significados de linearidade e RAE para analisar uma determinada proposta educacional.

Palavras-chave: Abordagem ontosemiótica. Raciocínio Algébrico. Linearidade.

\section{Introducción}

El Razonamiento Algebraico Elemental (RAE) tiene su centro de atención en los procesos de generalización y formalización que, tal como señalan Godino et al. (2014), siendo rasgos de dicho razonamiento el formalizar y generalizar patrones, identificar regularidades, y desarrollar prácticas matemáticas que involucren objetos como las relaciones binarias, operaciones, funciones y estructuras, fundamentalmente.

EI RAE se ha empleado en diversos trabajos de investigación desarrollados en el marco del Enfoque Ontosemiótico, centrados principalmente en identificar los conocimientos didáctico matemáticos de maestros de primaria en relación a este razonamiento (GODINO et al., 2015; TIAN; GIACOMONE y GODINO, 2020). También se han realizado investigaciones con estudiantes, como el de Burgos y Godino (2019), para desarrollar el razonamiento algebraico en estudiantes de quinto curso de educación primaria (10-11 años). Mientras que, en un trabajo, Aké y Godino (2018), se discute si las tareas de un libro de texto de primaria presentan rasgos que promuevan el pensamiento algebraico y, además, se proponen criterios que permiten discernir entre actividades matemáticas no algebraicas, proto-algebraicas y algebraicas. Finalmente, en el trabajo de Castro; Martínez Escobar y Pino-Fan (2017) se emplean los niveles de algebrización para analizar tareas propuestas en una colección de cinco libros de texto, usados en la primaria y se concluye que, si bien estos permiten predecir cierta actividad algebraica, las prácticas que pueden desarrollar los niños son muy diversas.

Como resultado de investigaciones como las descritas y de otras en las que se identifica el potencial que tienen determinadas tareas para desarrollar el razonamiento algebraico (GAITA y WILHELMI, 2019; BURGOS y GODINO, 2019) se puede observar que el RAE ha tomado relevancia en los últimos años y los resultados de las discusiones sobre su significado se ven hoy reflejados en los cambios en la enseñanza del álgebra escolar. Así, varios sistemas educativos escolares han adoptado nuevas posturas respecto al álgebra; en particular en el Currículo Nacional de la Educación Básica (PERÚ, 2016) se plantea que, para ser competente en matemática se deben desarrollar cuatro competencias a lo largo de la formación básica regular (hasta los 16 años), siendo una de ellas la competencia Resuelve problemas de regularidad, equivalencia y cambio. Dicha competencia se define de la siguiente manera: "el estudiante logre caracterizar equivalencias y generalizar regularidades y el cambio de una magnitud con respecto a otra, a través de reglas generales" (PERÚ, 2016, p. 73). Sin 
embargo, aún no se han abordado las implicaciones del modelo de niveles del RAE sobre la planificación curricular, tal como señalan Godino y Burgos (2017).

Es así que la presente contribución tiene por objetivo valorar la propuesta de una institución educativa en términos del desarrollo del RAE, a partir del tratamiento que esta propone para la linealidad pues, tal como indican Palanch y Ferreira (2021), el análisis de los libros de texto o materiales educativos de una institución aún debe ampliarse, dada la importancia de estos recursos para la educación.

Concretamente, se plantea realizar un estudio longitudinal, a lo largo de toda la escolaridad básica, para analizar en qué medida la propuesta de los colegios peruanos Innova Schools contribuye a desarrollar el RAE. Para ello, se prestará especial atención a la forma en la que han sido organizadas las prácticas matemáticas que involucran los diferentes significados asociados a la noción de linealidad y en qué medida estas permitirían la evolución del RAE a lo largo de la formación escolar. Consideramos que esto representará un aporte, ya que integrará nociones del EOS para analizar el significado pretendido en una institución en términos de si este genera prácticas potenciales que demanden cada vez mayores rasgos de algebrización.

\section{Elementos teóricos y metodológicos considerados en la investigación}

Para definir significados para la linealidad nos apoyamos en las investigaciones de Acosta (2011), en donde se establece la relación entre la proporcionalidad y la linealidad, en el trabajo de Burgos y Godino (2020), que presentan los diversos significados de la proporcionalidad, los cuales permitirían que emerjan distintos procesos y objetos algebraicos asociados, como la función lineal, en la investigación de Escudero (2017), que propone un significado de referencia para la función lineal y afín, así como en el aporte de Sánchez (2016), donde se señala que las tareas con patrones lineales (gráficos y numéricos) contribuyen a la consolidación de las concepciones de función lineal y afín.

En el EOS se considera que el significado de un objeto matemático está ligado a las prácticas asociadas a él, entendiendo por práctica matemática a toda actuación que realiza alguien al resolver un problema (GODINO; BATANERO y FONT, 2007). Tal como señalan Godino; Batanero; Burgos y Gea (2021), se aplica la herramienta configuración ontosemiótica de prácticas, objetos y procesos, en este caso, en su versión epistémica (significados institucionales) para abordar los problemas de la naturaleza de los objetos matemáticos y su conocimiento.

Es así que, a partir de los trabajos mencionados, proponemos un significado de referencia para la linealidad, considerando cada uno de los seis objetos primários (situaciones, lenguajes, conceptos, procedimientos, proposiciones y argumentos). Así, prestando especial atención a las situaciones 


\section{revemop}

problemas que involucran las nociones de razón, proporción, proporcionalidad directa, patrones lineales y función lineal, consideraremos los siguientes significados de la linealidad:

Significado informal: En este significado se deben trabajar situaciones que permitan desarrollar patrones de percepción y de cambio. En este significado no contempla aún nociones matemáticas de forma estricta, por lo tanto, el razonamiento es aún informal y apela al conocimiento propio del estudiante.

Significado aritmético: Este significado, previo a la proporcionalidad, representa el primer contacto con las nociones de magnitud, proporción y razón unitaria (razón constante de cambio), aunque sin hacer explícitos estos nombres. Las situaciones se caracterizan por el uso de operaciones aritméticas (multiplicación y división) para su solución y por la comparación de dos magnitudes.

Significado proporcional: En este significado se trabajan nociones propias de las magnitudes directamente proporcionales: proporción, fracciones equivalentes y constante de proporcionalidad (razón unitaria). Las situaciones están asociadas a determinar un valor en una proporción, al cálculo de la constante de proporcionalidad o razón unitaria, a completar tablas de proporcionalidad y encontrar relaciones de proporcionalidad entre magnitudes.

Significado funcional: Este significado representa una consolidación de la linealidad en la formación básica. Se trabaja de manera formal con la función lineal $f(x)=k x$ y con sus propiedades como son:

$$
f(a+b)=f(a)+f(b) \text { y } f(k a)=k f(a) .
$$

Las situaciones pueden presentarse en contextos gráficos, tabulares, conjuntos, analíticos y también en problemas sobre patrones lineales (secuencias gráficas y numéricas). Consideramos oportuno incluir las tareas con patrones lineales ya que estas demandarán encontrar una regla general de formación, que en este caso será una expresión lineal o lineal afín. Además, estas situaciones se caracterizan por encontrar la formalización en el lenguaje alfanumérico de la función lineal. Esta formalización debe darse a partir de reconocer que "un aumento constante en la variable $x$ " determina "un aumento constante en la variable $y$ ". Se presenta la Tabla 1 que muestra los objetos primarios asociados a cada significado de la linealidad en la educacion básica. 
Tabla 1: Objetos primarios de los significados de la linealidad

\begin{tabular}{|c|c|c|c|c|c|}
\hline $\begin{array}{l}\text { Significado de } \\
\text { la linealidad }\end{array}$ & Lenguajes & Conceptos & Procedimientos & Proposiciones & Argumentos \\
\hline Informal & Gráfico, verbal. & Ausencia & Ausencia & $\begin{array}{l}\text { A más..., más... } \\
\text { A menos..., } \\
\text { menos... }\end{array}$ & $\begin{array}{l}\text { Relacionados a la } \\
\text { experiencia del } \\
\text { estudiante. }\end{array}$ \\
\hline Aritmético & $\begin{array}{l}\text { Gráfico, verbal, } \\
\text { numérico. }\end{array}$ & $\begin{array}{l}\text { Cantidad, } \\
\text { razón, } \\
\text { figuras } \\
\text { semejantes }\end{array}$ & $\begin{array}{l}\text { Operaciones de división y } \\
\text { multiplicación. }\end{array}$ & $\begin{array}{l}3 \text { de ... es a } 12 \text { de } \\
\ldots, \text { como } 5 \text { de ... es } \\
\text { a } 20 \text { de... }\end{array}$ & $\begin{array}{l}\text { Si una cantidad } \\
\text { aumenta, } \\
\text { entonces la otra } \\
\text { también. }\end{array}$ \\
\hline Proporcional & $\begin{array}{l}\text { Verbal, } \\
\text { numérico, } \\
\text { tabular, } \\
\text { simbólico literal }\end{array}$ & $\begin{array}{l}\text { Cantidad, } \\
\text { magnitud, } \\
\text { razón, } \\
\text { proporción. }\end{array}$ & $\begin{array}{l}\text { Reducción a la unidad, uso } \\
\text { de proporciones, uso de } \\
\text { tablas de proporcionalidad, } \\
\text { regla de tres. }\end{array}$ & $\begin{array}{ll}\text { Dos pares } & \text { de } \\
\text { magnitudes } & \text { en } \\
\text { proporción } & \\
\text { determinan } & \text { una } \\
\text { igualdad. } & \end{array}$ & $\begin{array}{l}\text { Si dos } \\
\text { magnitudes son } \\
\text { directamente } \\
\text { proporcionales y } \\
\text { una dismininuye } \\
\text { la otra también. Y } \\
\text { viceversa }\end{array}$ \\
\hline Funcional & $\begin{array}{l}\text { Gráfico, verbal, } \\
\text { tabular, } \\
\text { simbólico literal }\end{array}$ & $\begin{array}{l}\text { Dominio, } \\
\text { rango, } \\
\text { pendiente, } \\
\text { función } \\
\text { lineal, } \\
\text { función afín }\end{array}$ & $\begin{array}{l}\text { Reconocer } \quad \text { cambios } \\
\text { constantes, establecer } \\
\text { relaciones } \\
\text { proporcionalidad como una } \\
\text { relación funcional lineal, } \\
\text { expresar la función } f(x)= \\
k x \text {. }\end{array}$ & $\begin{array}{l}\text { La función lineal es } \\
\text { homogénea, aditiva } \\
\text { y monótona. }\end{array}$ & $\begin{array}{l}\text { Un aumento } \\
\text { constante en la } \\
\text { variable "x" } \\
\text { determina } \\
\text { aumento un } \\
\text { constante en la } \\
\text { variable "y". }\end{array}$ \\
\hline
\end{tabular}

Fuente: Elaboración propia.

De otro lado, Godino et al. (2014) y Godino et al. (2015) amplian la definición de niveles de razonamiento algebraico de las prácticas matemáticas escolares para la primaria y secundaria; para ello, identifican los procesos y objetos involucrados inmersos en las prácticas consideradas como algebraicas. A continuación, una breve descripción de los niveles considerados para esta investigación:

Nivel 0: Ausencia de álgebra. Objetos extensivos (particulares) expresados en un lenguaje natural. Es posible la aparición de algún símbolo, esto no implica la generalización.

Nivel 1: Intervienen objetos intensivos (generales) en un lenguaje natural, numérico o gestual. En tareas funcionales la generalidad se expresa en lenguaje no alfanumérico.

Nivel 2: Uso de variables para representar un objeto intensivo. Se resuelven ecuaciones de la forma $A x \pm B=C$ y en tareas funcionales la generalización se expresa en lenguaje alfanumérico, pero aún no se realizan transformaciones a dichas expresiones.

Nivel 3: La generalidad se formaliza en expresiones alfanuméricas y se realizan tratamientos para encontrar expresiones equivalentes (transformaciones). Se resuelven ecuaciones de la forma $A x \pm$ $B=C x \pm D$. Nivel consolidado de algebrización.

Nivel 4: Implica el empleo de parámetros, como valores constantes que no cambian y permiten representar familias de funciones y ecuaciones. 
Es importante señalar que los niveles propuestos representan una caracterización general para cualquier práctica matemática; es por ello que, como parte de esta investigación, nos proponemos adaptar estos niveles a situaciones asociadas a la linealidad.

Posteriormente, se identificará el significado pretendido por la institución educativa Innova Schools para la linealidad y se valorará si dicha propuesta propicia un desarrollo progresivo del razonamiento algebraico, teniendo como foco de atención la linealidad. Para ello, se tendrá en cuenta el trabajo de Castro; Martínez-Escobar y Pino-Fan (2017), en donde se analizó un conjunto de libros, asignando a los problemas propuestos el nível 0,1,2 y 3 de algebrización, en función a la práctica matemática que ellos demandaban. En particular, nos centraremos en reconocer aquellas situacionesproblemas con potencial para construir los diferentes significados de la linealidad y desarrollar el RAE.

A continuación, se muestran los resultados obtenidos en cada etapa.

\section{Niveles de razonamiento algebraico elemental y su relación con los significados de linealidad}

En el apartado anterior mostramos que cada significado de la linealidad pone en juego distintos procesos y objetos algebraicos; en base a ello realizamos una adaptación de los niveles del RAE para la linealidad. Es decir, reconocemos qué procesos algebraicos emergen al resolver situaciones propias de cada significado y se les asocia un nivel del RAE.

\section{NIVEL 0}

Este nivel está asociado al significado informal, aritmético y a determinadas situaciones de patrones lineales. El razonamiento para reconocer semejanzas o situaciones proporcionales no se sustenta en argumentos formales. La actividad matemática se limita a operaciones aritméticas (multiplicación y división) con cantidades particulares (extensivos) mediante un lenguaje natural, numérico, icónico o gestual al hallar valores desconocidos.

En tareas de patrones lineales, la determinación de términos particulares (extensivos) se da mediante operaciones numéricas en un lenguaje natural, numérico, icónico o gestual. Pueden aparecer símbolos, pero estos surgen para representar a un objeto extensivo y no supone una manipulación simbólica. En este nivel no se realizan generalizaciones, formalizaciones 0 transformaciones.

\section{NIVEL 1}

Este nivel está asociado al significado aritmético, proporcional y también a algunas situaciones de patrones lineales. Aparecen procesos como la generalización, al hallar una razón unitaria en 
situaciones de proporcionalidad, aunque sin el uso explícito de un lenguaje alfanumérico. No se presentan los procesos de formalización o transformación.

Se hace uso de la igualdad como equivalencia al reconocer fracciones equivalentes:

$$
\frac{a}{b}=\frac{c}{d}
$$

La operación de producto en aspa de la equivalencia determinada se sustenta en las propiedades de los números naturales. Esto se da en un lenguaje natural, numérico, icónico o gestual.

En tareas de patrones lineales, la determinación de términos particulares se da mediante el reconocimiento de un patrón a partir de recuentos explícitos hechos mediante el cálculo numérico 0 representaciones gráficas, sin uso del lenguaje alfanumérico. Esto se da para términos cercanos a los dados en la sucesión, por lo que aún no se determina el patrón general de formación.

\section{NIVEL 2}

En este nivel aparecen procesos de generalización y formalización, ya que se usan expresiones simbólicas - literales para determinar un valor desconocido en una proporción. También, se resuelven ecuaciones de la forma $A x=B$. Esto aparece al expresar una razón unitaria en un lenguaje alfanumérico, por ejemplo " $6 n$ " y al igualar dos fracciones equivalentes, generando una

ecuación proporcional $x=\frac{b c}{d}$. El análisis se da en un lenguaje natural, numérico, icónico o gestual al principio, luego se trabaja haciendo uso de expresiones simbólico - literales, aunque sin hacer transformaciones a las expresiones halladas.

En patrones lineales, debe darse la generalización y formalización al determinar una relación entre el término y el lugar que ocupa en la secuencia. Esto debe expresarse de forma simbólico - literal, aunque aún no se hacen transformaciones en este registro.

\section{NIVEL 3}

Este nivel es característico del significado funcional, son inherentes los procesos de generalización, formalización y transformación. La proporcionalidad debe consolidarse como una relación funcional entre dos variables que se expresa formalmente como la función lineal $f(x)=k x$, donde $k$ es un valor constante. Así también aparece la función afín $g(x)=k x+b$, donde $k$ y $b$ son constantes. Además, en este nivel se hacen uso de las propiedades de la linealidad:

$$
f(x+y)=f(x)+f(y) \text { y } f(k x)=k f(x),
$$

y la monotonía de las funciones lineales, como una formalización de lo trabajado en el significado informal llevado al significado funcional (un aumento en la variable $x$ implica un aumento en la 
variable $y$ ). En situaciones funcionales o previas a estas, como el caso de problemas de patrones lineales, se realizan transformaciones a las expresiones halladas; es decir, se resuelven ecuaciones de la forma $A x \pm B=C x \pm D$.

\section{NIVEL 4}

Este nivel es asociado a las situaciones del significado funcional, con la diferencia de que el grado de generalidad que se requiere es mayor.

El parámetro puede ser abordado en sus diferentes significados. Esto es, como un valor constante que no cambia: se asigna un valor al parámetro " $a$ " en la función lineal $f(x)=a x$, donde se estudia la variación de la función para cada valor de x. O como un valor cambiante: se interpreta cómo es el cambio de la función $f(x)=a x$ al cambiar el parámetro " $a$ ", esto es, se estudian diferentes funciones, analizando el efecto que produce variar el parámetro. $O$ como una incógnita: se resuelven ecuaciones con parámetros al igualar dos expresiones de funciones lineales. 0 también como generalizador, lo que requiere emplear parámetros para representar familias de funciones lineales.

A modo de síntesis, se presenta la Tabla 2, en donde se relacionan los significados y los rasgos de los niveles de algebrización que demandan las tareas asociadas a ellos.

Tabla 2: Nivel y significados de la linealidad

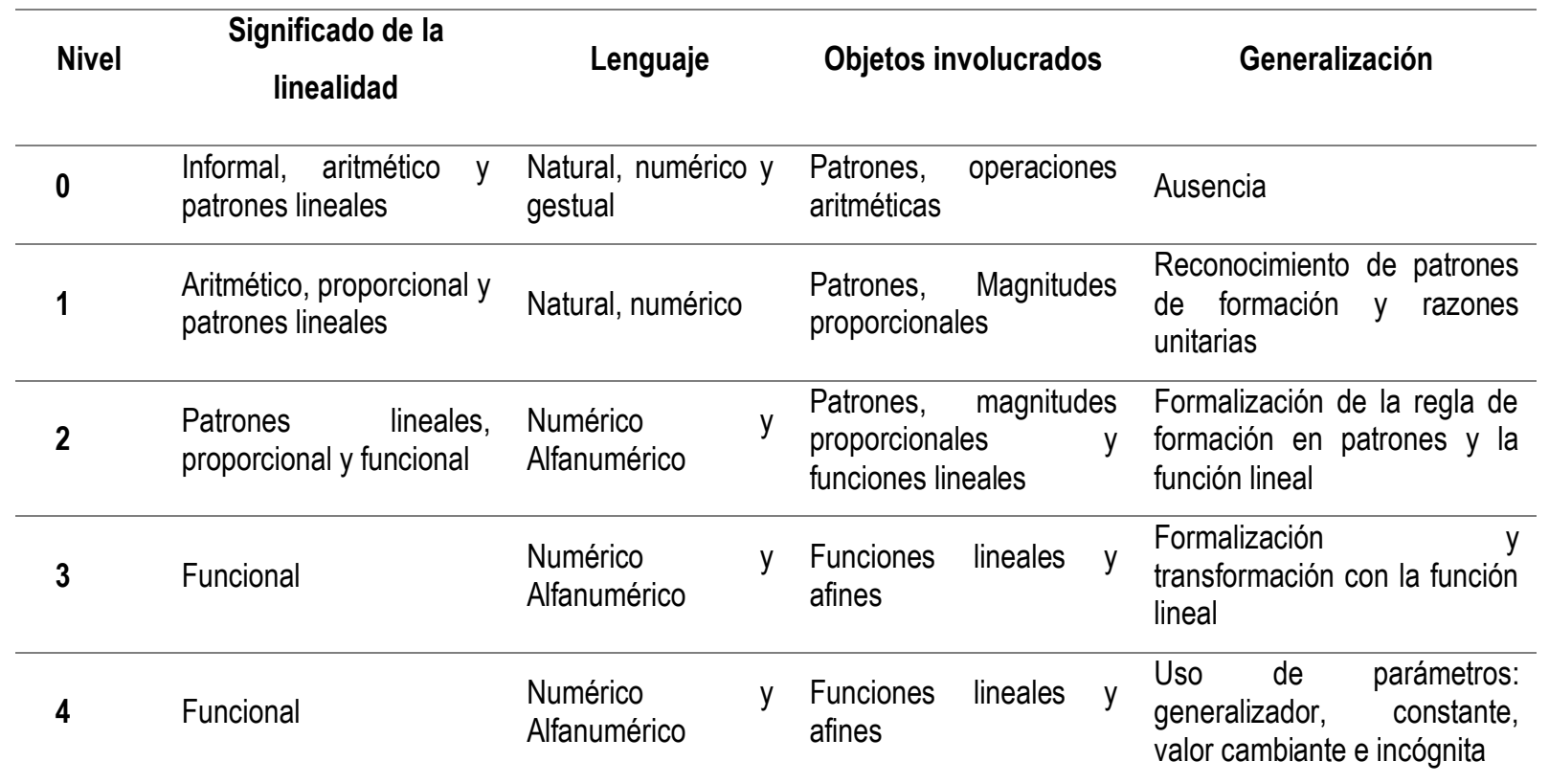

Fuente: Elaboración propia.

Estos niveles serán utilizados para valorar la propuesta educativa de la institución Innova Schools, red de colegios peruanos privados que se fundó en el año 2005 y que actualmente cuenta con 63 sedes en Lima y provincias. El área curricular de matemáticas se enmarca en los lineamientos 
generales que brinda el Currículo Nacional de la Educación Básica (PERÚ, 2016), como lo son las competencias y los estándares de aprendizaje.

Se analizará cada situación problema considerada en los diversos materiales empleados durante la formación básica (primaria y secundaria). A cada problema se le asociarán significados de la linealidad y rasgos de las prácticas matemáticas que demandarían su resolución. En la siguiente sección se mostrarán los resultados de esta valoración.

\section{Valoración de la institución Innova Schools respecto a la evolución del RAE}

Las competencias son definidas como "la facultad que tiene una persona de combinar um conjunto de capacidades a fin de lograr un propósito específico em una situación determinada, actuando de manera pertinente y con sentido ético" (PERÚ, 2016, p. 29). Para el área de matemática se determinan cuatro competencias:

- Resuelve problemas de cantidad

- Resuelve problemas de regularidad, equivalência y cambio.

- Resuelve problemas de forma, movimento y localización

- Resuelve problemas de gestión de datos e incertidumbre

Los estándares de aprendizaje son definidos como descripciones del nivel de desarrollo de los estudiantes, desde el nível primário al secundario, en una determinada competencia (PERÚ, 2016, p. 36). Estas descripciones de desarrollo están graduadas en 8 niveles.

Pese a que la propuesta curricular de la institución Innova Schools se enmarca dentro de los lineamientos generales del Currículo Nacional de la Educación Básica (PERÚ, 2016) esta institución propone desarrollar una sola competencia matemática en base a cuatro organizadores matemáticos. Estos organizadores son semejantes a las cuatro competencias antes indicadas. En la Tabla 3 se describen los cuatro organizadores: 


\section{Organizador}

Resuelve problemas de

\section{Descripción}

Referido al tratamiento de los conjuntos numéricos en sus distintas formas de

Cantidad representación, sus gráficos y operaciones; la medición de magnitudes como tiempo, masa y temperatura. Es decir, se trata de la cuantificación de los objetos del mundo real, empleando distintos sistemas de medida.

Referido a la identificación, representación, análisis y modelación del cambio en los

Regularidad, equivalencia cambio

y en los distintos fenómenos y eventos y su generalización. Pueden ser representadas de

muy diversas maneras: verbalmente, de manera numérica, simbólica o gráficamente. Las relaciones matemáticas pueden ser establecidas echando mano a nociones como las funciones, ecuaciones o desigualdades.

Movimiento,
forma
localización

La forma es un término que posee un vínculo muy estrecho con la geometría, pero en este caso va mucho más allá de estos significados y métodos. Este organizador está y relacionado además con las formas reales, su representación gráfica y analítica y la interpretación de la información visual. Asimismo, está referido a los patrones geométricos, la ubicación, trayectoria y las transformaciones espaciales.

\section{Gestión de datos e incertidumbre}

Referido a estos dos tópicos muy relacionados. Estos fenómenos son la materia de estudio de la estadística y de la probabilidad, respectivamente. Los conceptos y actividades que son importantes en esta área son la recolección de datos, el análisis e interpretación de datos y sus representaciones. Asimismo, la probabilidad y la inferencia y el tratamiento de la incertidumbre.

\section{Fuente: Elaboración propia.}

Para el desarrollo de esta investigación se solicitó acceso a documentos restringidos de la institución, tales como el Diseño curricular del área de matemáticas (INNOVA SCHOOLS, 2019) y a los materiales empleados en las sesiones de clase relacionadas con la linealidad, de los grados 1 al 10 (desde los 6 años hasta los 15 años). Se identificaron las sesiones de aprendizaje en donde se abordó algún significado de la linealidad a partir de la revisión del Diseño curricular del Área de Matemática de la institución; además, en la plataforma virtual del Teacher Resource Center se encontraron las fichas de ejercicios y problemas a usarse en las sesiones de cada grado. Estos documentos han sido fundamentales para valorar la propuesta educativa en términos del desarrollo del RAE.

Dado que la propuesta de la institución también hace uso de estándares de aprendizaje, los cuales miden el avance académico de los estudiantes a lo largo de la formación básica, se identificaron los estándares que guardaban relación con los diferentes significados de la linealidad. Luego, teniendo en cuenta los estándares seleccionados, se identificó en qué sesiones se abordaron situaciones relacionadas con dichos estándares en los grados mencionados. Se clasificaron las situaciones problema presentados en cada sesión, de acuerdo a los diversos significados de linealidad. 
Finalmente, se analizaron las situaciones problema presentadas para determinar en qué medida las prácticas matemáticas que estas situaciones demandaban, mostraban rasgos asociados a distintos niveles de razonamiento algebraico.

\subsection{Estándares seleccionados del primer al décimo grado}

A continuación, se presentan los estándares identificados en la propuesta curricular que abordan algunos significados de la linealidad, señalando a qué grado corresponden. Esta identificación permitió reconocer las sesiones en donde se abordaban los problemas sobre la linealidad. A modo de ejemplo, presentamos las descripciones de algunos de los estándares en donde pueden identificarse los objetos y procesos considerados como algebraicos.

En segundo grado de educación primaria se describe el estándar MAT.2.21 como sigue: "Resuelve situaciones que demandan crear, continuar, interpretar, identificar y graficar patrones geométricos y numéricos con números naturales de hasta dos cifras" (INNOVA SCHOOLS, 2019, p. 15).

Mientras que en tercer grado de primaria se propone el estándar MAT.3.14: "Resuelve situaciones problemáticas con números naturales y de una o más etapas, en el campo aditivo (...); en el campo multiplicativo (proporcionalidad simple, producto de medidas, arreglos), así como problemas que involucran al campo aditivo y multiplicativo" (INNOVA SCHOOLS, 2019, p. 16).

En el cuarto grado se plantea el estándar MAT.4.23: "Resuelve situaciones con material concreto que demanden identificar y explicar las relaciones de cambio entre dos magnitudes" (Innova Schools, 2019, p.17).

En el quinto grado se plantea el estándar MAT.5.23: "Resuelve situaciones de cambio que demanden establecer relaciones de proporcionalidad directa y relaciones de equivalencia entre unidades de medida de una misma magnitud, usando tablas o el plano cartesiano" (INNOVA SCHOOLS, 2019, p. 18).

En sexto grado, MAT.6.23: "Resuelve situaciones susceptibles de ser modeladas mediante relaciones de proporcionalidad simple (directa e inversa), usando tablas, el plano cartesiano y expresiones simbólicas" (INNOVA SCHOOLS, 2019, p. 19).

En séptimo grado se propone el estándar MAT.7.24, que señala que los estudiantes deben ser capaces de hacer lo siguiente: "Resuelve situaciones susceptibles de ser modeladas mediante relaciones de proporcionalidad simple (directa e inversa) o las funciones lineales y afines, usando tablas, el plano cartesiano y expresiones simbólicas" (INNOVA SCHOOLS, 2019, p. 19). 
Tabla 4. Resumen de estándares asociados a la linealidad en primaria y secundaria

\begin{tabular}{|c|c|c|}
\hline Grado & Organizador & $\begin{array}{l}\text { Estandar } \\
\text { asociado }\end{array}$ \\
\hline \multirow{2}{*}{1} & Regularidad, equivalencia y cambio & MAT.1.21 \\
\hline & Forma, movimiento y localización & MAT.1.33 \\
\hline \multirow{3}{*}{2} & Regularidad, equivalencia y cambio & MAT.2.21 \\
\hline & Cantidad & MAT.2.14 \\
\hline & Forma, movimiento y localización & MAT.2.31 \\
\hline \multirow{4}{*}{3} & Regularidad eguivalencia y cambin & MAT.3.21 \\
\hline & 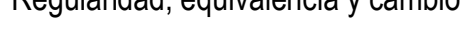 & MAT.3.23 \\
\hline & Cantidad & MAT.3.14 \\
\hline & Forma, movimiento y localización & MAT.3.31 \\
\hline \multirow{3}{*}{4} & Regularidad equivalencia v cambio & MAT.4:21 \\
\hline & Regularıaad, equivaiencla y camoio & MAT.4.23 \\
\hline & Forma, movimiento y localización & MAT.4.31 \\
\hline \multirow{3}{*}{5} & Reqularidad equivalencia y cambio & MAT.5.21 \\
\hline & Reguraridad, equivarencra y camoio & MAT.5.23 \\
\hline & Forma, movimiento y localización & MAT.5.33 \\
\hline \multirow{3}{*}{6} & Reoularidad equivalencia v cambin & MAT.6.21 \\
\hline & Reguraridad, equivarencra y camoio & MAT.6.23 \\
\hline & Forma, movimiento y localización & MAT.6.33 \\
\hline \multirow{2}{*}{7} & Regularidad, equivalencia y cambio & MAT.7.24 \\
\hline & Forma, movimiento y localización & MAT.7.32 \\
\hline \multirow{2}{*}{8} & \multirow{2}{*}{ Regularidad, equivalencia y cambio } & MAT.8.23 \\
\hline & & MAT.8.24 \\
\hline \multirow{2}{*}{9} & \multirow{2}{*}{ Regularidad, equivalencia y cambio } & MAT.9.21 \\
\hline & & MAT.9.23 \\
\hline 10 & Regularidad, equivalencia y cambio & MAT.10.22 \\
\hline
\end{tabular}

\subsection{Análisis de las situaciones problema en términos del desarrollo del RAE.}

En esta sección clasificamos las situaciones - problemas que permiten el desarrollo del RAE en lo que respecta a la linealidad, de acuerdo a los significados elaborados. Dado que un mismo significado es abordado en diferentes grados, el análisis se centró en determinar si al abordar situaciones asociadas a un mismo significado en diferentes grados, estas demandan diferentes niveles del RAE

Evolución del RAE en situaciones asociadas al significado informal

Las situaciones asociadas a este significado aparecen en el 1er y 5 to grado de la primaria. Estas tareas tienen la finalidad de reconocer figuras semejantes por sus características (1er grado) y cómo es el cambio de una figura respecto a otra (5to grado). En ambos casos no se requiere de 
ningún proceso algebraico para dar solución a los problemas, por lo que no se determina evolución del RAE en tareas asociadas a este significado.

Figura 1: Situaciones del significado informal

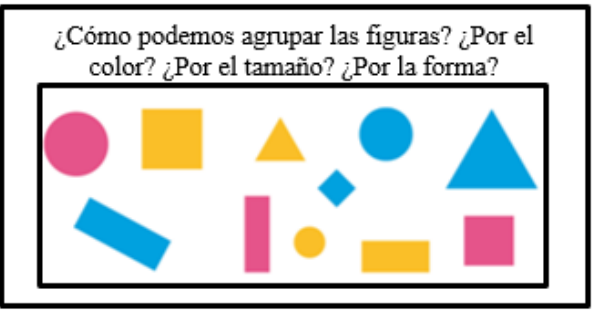

Situación propuesta en el 1er grado de primaria

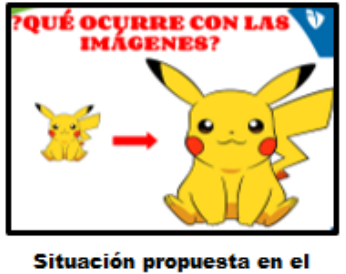

5to grado de primaria

Fuente: TRCVIVO.INNOVASCHOOLS (2019).

\section{Evolución del RAE en situaciones asociadas al significado aritmético}

Este significado aparece en ambos niveles: primaria (2do, 3er, 4to, 5to y 6to grado) y secundaria (1er y 2 do grado). A continuación, unos ejemplos de situaciones en dichos grados:

Figura 2: Situaciones del significado aritmético

7. Jaime elabora un juguete para niños usando un par de botellas de
plástico. ¿Cuántos juguetes podrá elaborar con 24 botellas de plástico?

Situación propuesta en el 2do grado

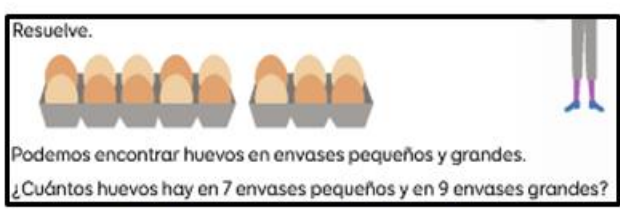

Situación propuesta en el 3er grado

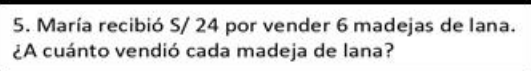

Situación propuesta en el 4to grado

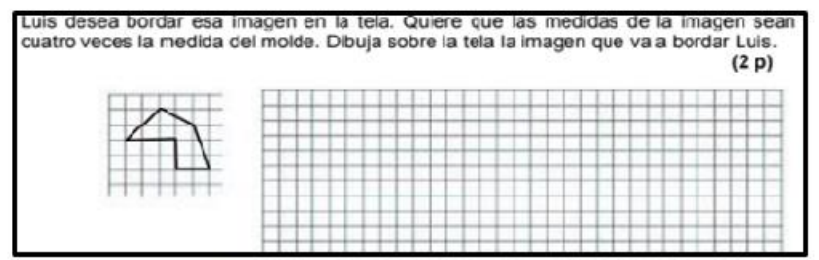

Situación propuesta en el 8vo grado (2do de secundaria)

Fuente: TRCVIVO.INNOVASCHOOLS (2019)

En las situaciones propuestas de los primeros grados la actividad matemática se centra en encontrar un valor desconocido, mediante operaciones aritméticas, a partir de la relación de dos magnitudes. Algo similar ocurre en la situación del nivel secundario, se demanda multiplicar las dimensiones de una figura. Dado que en estas situaciones se requiere del cálculo aritmético se determina ausencia de álgebra (nivel 0 del RAE).

\section{Evolución del RAE en situaciones asociadas al significado proporcional}

Este significado aparece en ambos niveles: primaria (3ro, 4to, 5to y 6to grado) y secundaria (1er y 2do grado). A continuación, unos ejemplares de dichos grados: 
Figura 3: Situaciones del significado proporcional

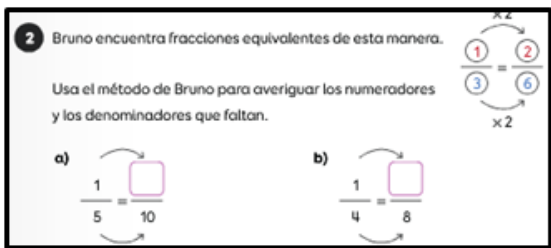

Situación propuesta en el 3er grado

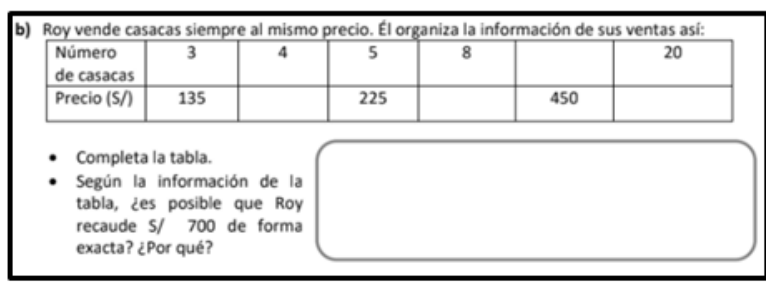

Situación propuesta en el 5 to grado

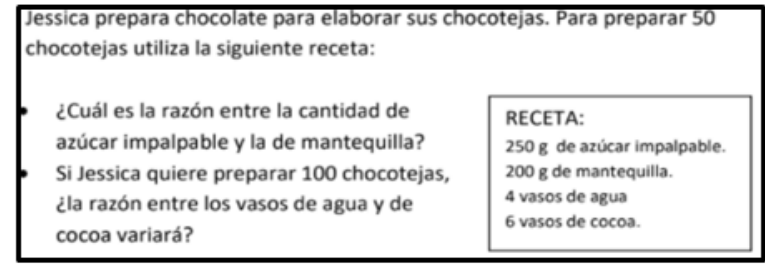

Situación propuesta en el $7 \mathrm{mo}$ grado (1ro de secundaria)

Dos magnitudes $\mathrm{M}$ y $\mathrm{N}$ son directamente proporcionales (DP). Cuando $\mathrm{M}$ es

igual a $8, N$ es igual 24. ¿Cuál será el valor de $M$ cuando $N$ sea 27 ?

Situacón propuesta en el 8vo grado (2do de secundaria)

Fuente: TRCVIVO.INNOVASCHOOLS (2019)

En este significado de la linealidad sí se evidencia un desarrollo del RAE. La situación del 3er grado se limita a un cálculo aritmético (nivel 0), pero en la situación del 5to grado se observa una actividad que demanda relacionar dos magnitudes y con ello hallar valores desconocidos con ecuaciones de la forma $x=\frac{a \times b}{c}$ (nivel 2). Esto es más evidente en la actividad del 8vo grado. Por otro lado, la actividad del 7 mo grado demanda encontrar la razón unitaria entre dos magnitudes y ver cómo esta varía, esta razón unitaria es una forma de generalizar sin hacer uso de un lenguaje alfanumérico (nivel 1).

Evolución del RAE en situaciones asociadas al significado en patrones lineales

Este significado aparece en ambos niveles: primaria (1er, 2do, 3er, 4to y 6to grado) y secundaria (2do y 3er grado). A continuación, presentamos unos ejemplos de atividades para dichos grados: 
Figura 4: Situaciones del significado en patrones lineales

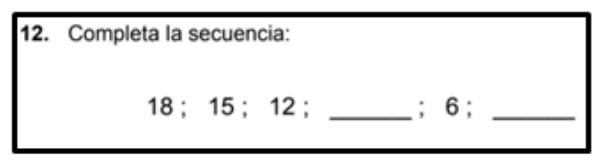

Situación propuesta en el 1er grado

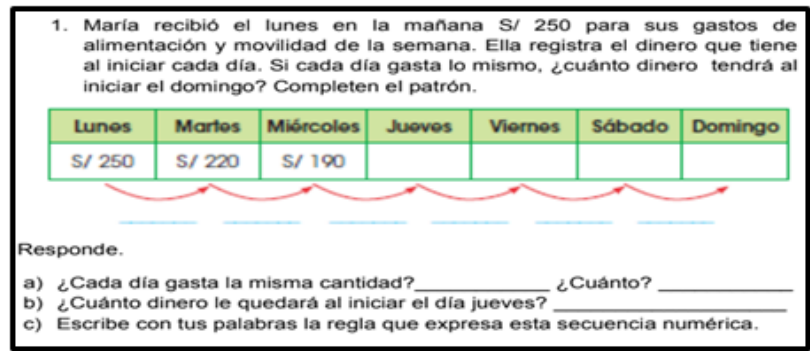

Situación propuesta en el 4to grado

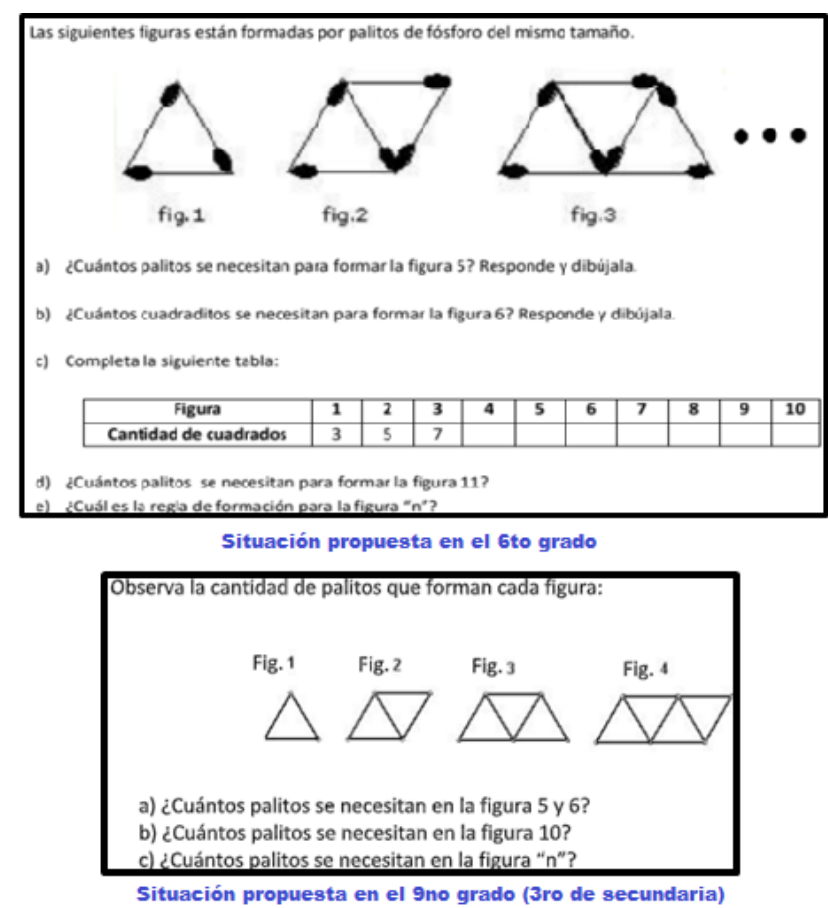

Fuente: TRCVIVO.INNOVASCHOOLS (2019)

En este tipo de situaciones también se evidencia un desarrollo del RAE. En las situaciones del 1er grado, similares a las del 2 do y 3 er grado, la actividad se centra en hallar valores cercanos de secuencias numéricas (nivel 0). Es a partir de las situaciones del 4to grado en que se observa un aumento en la demanda de la tarea, es así que dicha situación requiere que se exprese la regla de formación en un lenguaje natural (nivel 1). Niveles 2 y 3 del RAE se observa en la actividad del 6to grado, en la que explícitamente se pide hallar una expresión para un término " $n$ ". Así también sucede en la actividad del 3er grado de secundaria.

\section{Evolución del RAE en situaciones asociadas al significado funcional}

Este significado es característico del nivel secundario (1er, 2do, 3er y 4to grado), pero también evidenciamos su uso en el 4to grado del nivel primaria. A continuación, presentamos algunos ejemplos de situaciones pertenecientes a dichos grados: 
Figura 5: Situaciones del significado funcional en primaria

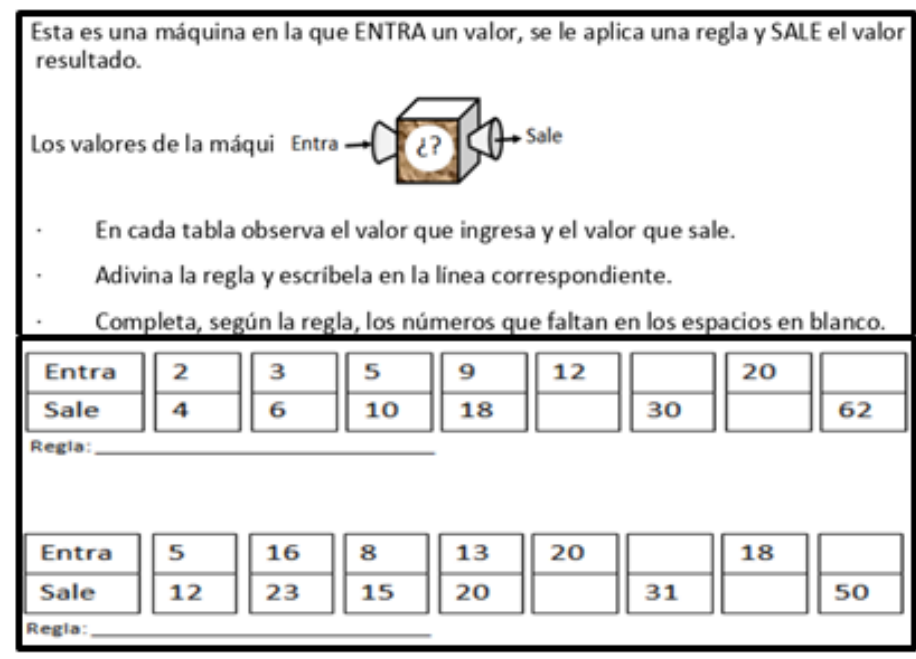

Situación propuesta en el 4to grado de primaria

Fuente: TRCVIVO.INNOVASCHOOLS (2019)

La situación mostrada centra la actividad matemática en completar tablas de proporcionalidad y reconocer la regla de formación en un lenguaje natural (nivel 1). Esta situación propone términos de funciones, como valor de entrada y salida, de una forma más familiar a los estudiantes.

Figura 6. Situaciones del significado funcional en secundaria

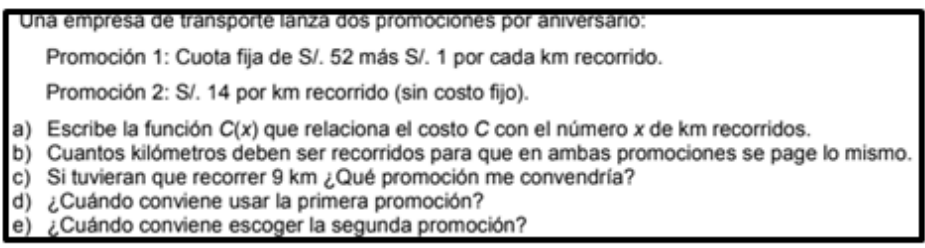

Situación propuesta en el 8vo grado (2do de secundaria)

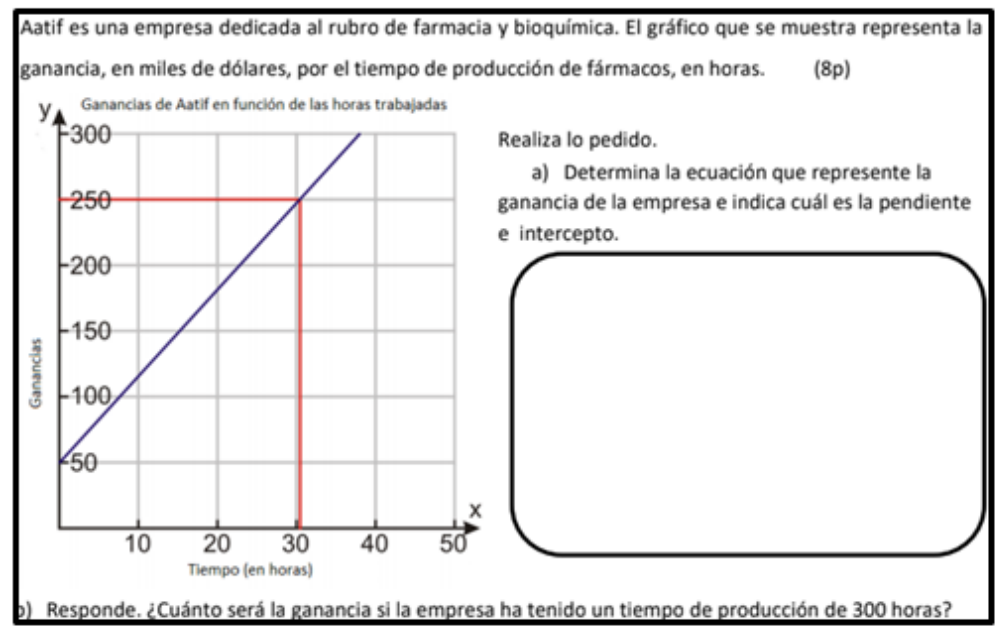

Situación propuesta en el 9no grado (3ro de secundaria
Sea la funciṓn $f(x)=-6 x-5$.
¿Cuál o cuáles de las siguientes
afirmaciones son verdaderas?
L. La función es decreciente
II. La fecta corres pondiente a la función
interseca al eje Yen el punto $(-5 ; 0)$.
III. El punto (1; -11) pertenece a la
funciōn.
a) Solo I
b) Solo II
c) Solo III
c) Iy III
e) II y III

Situación propuesta en el $10 \mathrm{mo}$ grado (4to de secundaria)

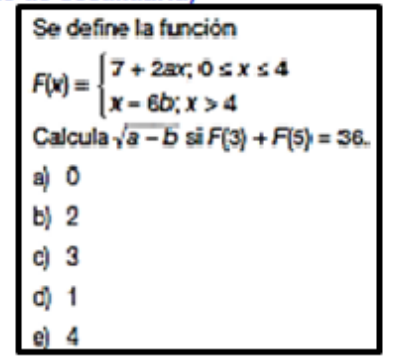

Situación propuesta en el $10 \mathrm{mo}$ grado (4to de secundaria) 
Las situaciones para el nivel secundaria, en general, demandan encontrar la regla que exprese la relación entre dos magnitudes haciendo uso de un lenguaje alfanumérico (nivel 2 del RAE). Esto se evidencia en las situaciones del 8vo y 9 no grado, de forma similar ocurren el 7 mo grado. Además, en algunos casos, como en la actividad del 8vo grado, la actividad demanda encontrar para qué valor de " $x$ " las funciones son iguales, por lo que se resuelven ecuaciones de la forma $A x+B=C x+D$ (Nivel 3 del RAE).

Es en el 4to grado de secundaria en que la actividad matemática deja de lado el hallar expresiones que generalicen la relación entre dos magnitudes, la actividad se centra en trabajar con expresiones en un lenguaje puramente algebraico. Las situaciones demandan reconocer si una función es creciente o decreciente, interpretar funciones por tramos, hallar pendientes y el trabajo con parámetros. Por lo que se determina un nivel 4 del RAE.

\section{Consideraciones finales}

Nuestro estudio tuvo por interés valorar una propuesta educativa en términos de si esta propicia la evolución del Razonamiento Algebraico en actividades asociadas a la noción de linealidad. Para ello, se consideraron los significados de la noción de linealidad para la formación básica. Este significado respondía a los elementos que surgen en toda práctica matemática y quedó caracterizado por cómo deben ser sus situaciones. Así también, se adaptaron y relacionaron los niveles del RAE a los significados propuestos para la noción de linealidad, a fin de poder valorar la práctica matemática que se desprende de trabajar las situaciones de dicha noción matemática.

Los resultados muestran que la propuesta de la institución Innova Schools trabaja los diferentes significados de la linealidad y, además, sí se propicia la evolución del RAE al trabajar situaciones asociadas a la linealidad. En el nivel primario la actividad se centra en los significados informal, aritmético y proporcional y en el nivel secundario en el significado funcional.

En relación a la evolución del RAE, es en los primeros grados de la primaria que la actividad algebraica es de nivel 0 y 1 , luego esta actividad aumenta hasta los niveles 2 y 3 en los grados posteriores de la primaria y en la secundaria la actividad matemática aumenta hasta el nivel 4.

Sin embargo, pese a reconocer una evolución del Razonamiento Algebraico consideramos que se debe ajustar la propuesta en los grados del nivel secundario ( $7 \mathrm{mo}, 8 \mathrm{vo}$ y $9 \mathrm{no})$, ya que muchas de las situaciones demandan la misma exigencia en términos de procesos y de la movilización de objetos involucrados en los tres grados. La gran mayoría de tareas en esos grados se centran en reconocer la 
regla de formación general, limitando la actividad a un nivel 2 y 3 del RAE. Es recién en el 10mo grado donde se identificó una tarea que demanda desarrollar prácticas matemáticas con rasgos del nivel 4.

Una valoración más completa de la propuesta educativa analizada en términos del desarrollo del razonamiento algebraico requiere considerar el significado implementado. Esto implica identificar cómo se emplean y gestionan las actividades previstas en los materiales que la institución provee en las prácticas matemáticas desarrolladas en el aula, esta es una tarea pendiente.

Siguiendo la propuesta de Carpes y Bisognin (2021), consideramos que los resultados obtenidos deberían formar parte de los conocimientos didácticos matemáticos de los docentes de matemáticas y ser discutidos en cursos de formación continua donde se discuta explícitamente las prácticas de enseñanza en torno a la linealidad.

Finalmente, consideramos que, así como este trabajo ha mostrado que es posible articular los niveles de razonamiento algebraico con un objeto particular como lo es la linealidad, queda pendiente realizar esta identificación con otros objetos matemáticos considerados como algebraicos.

\section{Reconocimiento:}

Este trabajo se desarrolló como parte del proyecto CAP Competencias didáctico-matemáticas del profesorado para el desarrollo del razonamiento algebraico, con el apoyo de la Pontificia Universidad Católica del Perú.

\section{Referencias}

ACOSTA, Juan Alberto. La noción de linealidad. Una aproximación epistemológica, cognitiva, didáctica y sociocultural. 2011. 267f. (Tesis doctoral). Instituto Politécnico Nacional, D.F. México.

AKÉ, Lilia; GODINO, Juan. Análisis de tareas de un libro de texto de primaria desde la perspectiva de los niveles de algebrización. Educación Matemática, v. 30, n. 2, p. 171-201, 2018.

BURGOS, María; GODINO, Juan. Emergencia del razonamiento proto-algebraico en tareas de proporcionalidad en estudiantes de primaria. Educación Matemática, v. 31, n. 3, p. 117-150, 2019.

BURGOS, María; GODINO, Juan. Modelo ontosemiótico de referencia de la proporcionalidad. Implicaciones para la planificación curricular en primaria y secundaria. AIEM, v. 18, p.1-20, 2020.

CARPES, P. P. G.; BISOGNIN, E. A Formação Continuada de Professores na perspectiva dos Conhecimentos Didáticos Matemáticos. Revemop, v. 3, p. e202111, 23 jun. 2021.

CASTRO, Walter; MARTíNEZ-ESCOBAR, John; PINO-FAN, Luis. Niveles de algebrización de la actividad matemática escolar: Análisis de libros de texto y dificultades de los estudiantes. REDIMAT, v. 


\section{6, n. 2, p. 164-191, 2017.}

ESCUDERO, Phamela. Identificación de conocimientos didáctico-matemáticos, en la faceta epistémica, del profesor de educación secundaria, sobre funciones lineales y cuadráticas. 2017. 100f. (Tesis de maestría). Pontificia Universidad Católica del Perú, Lima. Perú.

GAITA, Cecilia; WILHELMI, Miguel. Desarrollo del razonamiento algebraico elemental mediante tareas de recuento con patrones. Bolema, v.33, n. 63, p. 269-289, 2019.

GODINO, Juan; BATANERO, Carmen; FONT, Vicenç. The onto-semiotic approach to research in mathematics education. ZDM. The International Journal on Mathematics Education, v. 39, n. 1-2, p. 127-135, 2007.

GODINO, Juan; AKÉ, Lilia; GONZATO, Margherita; WILHELMI, Miguel. Niveles de algebrización de las prácticas matemáticas escolares. Implicaciones para la formación de maestros. Enseñanza de las Ciencias, v. 32, n. 1, p. 199-219, 2014.

GODINO, Juan; RIVAS, Hernán; ARTEAGA, Pedro; LASA, Aitzol; WILHELMI, Miguel. Ingeniería didáctica basada en el enfoque ontológico - semiótico del conocimiento y de la instrucción matemáticos. Recherches en Didactique des Mathématiques, v. 34, n. 3-4, p. 167-200, 2014.

GODINO, Juan; AKÉ, Lilia; CONTRERAS, Angel; DÍAZ, Carmen; ESTEPA, Antonio; BLANCO, Teresa; LACASTA, Eduardo; LASA, Aitzol; NETO, Teresa; OLIVERAS, Luisa; WILHLEMI, Miguel. Diseño de un cuestionario para evaluar conocimientos didáctico-matemáticos sobre razonamiento algebraico elemental. Enseñanza de las Ciencias, v. 33, n. 1, p. 127-150, 2015.

GODINO, Juan; NETO, Teresa; WILHELMI, Miguel; AKÉ, Lilia; ETCHEGARAY, Silvia; LASA, Aitzol. Niveles de algebrización de las prácticas matemáticas escolares. Articulación de las perspectivas ontosemiótica y antropológica. Avances de Investigación en Educación Matemática, v.8, p. 117-142, 2015.

GODINO, Juan; BURGOS, María. Perspectiva ontosemiótica del razonamiento algebraico escolar. En J.M. MUÑOZ-ESCOLANO; A. ARNAL-BAILERA; P. BELTRÁN-PELLICER; M. CALLEJO; y J. CARRILLO (Eds.), Investigación en Educación Matemática XXI (pp. 49-66). Zaragoza: SEIEM, 2017.

GODINO, Juan; BATANERO, Carmen; BURGOS, María; GEA, María. Uma perspectiva ontosemiótica dos problemas e métodos de pesquisa em educação matemática. Revemop, v. 3, p. e202107, 2021. DOI: https://doi.org/10.33532/revemop.e202107

INNOVA SCHOOLS. Diseño curicular del área de matemática. 2019.

PALANCH, W. B. DE L.; FERREIRA, R. B. O Enfoque Ontossemiótico: uma análise da geometria na coleção de livro didático de Matemática no Ensino Técnico Integrado ao Médio. Revemop, v. 3, p. e202113, 7 jul. 2021. DOI: https://doi.org/10.33532/revemop.e202113

PERÚ. Ministerio de Educación del Perú. Currículo Nacional de la Educación Básica. 224p., 2016. Disponible en: http://www.minedu.gob.pe/curriculo/pdf/curriculo-nacional-de-la-educacion-basica.pdf. Acceso en: 10 mar. 2021.

SÁNCHEZ, Diana. Conceptualización de la función lineal y afín: Una experiencia de aula. 2016. 125f. (Tesis de maestría). Universidad Distrital Francisco José de Caldas, Bogotá. Colombia. 


\section{revemop}

TIAN, Guoping; GIACOMONE, Belén; GODINO, Juan. In service teacher's Didactic-Mathematical Knowledge on Elementary Algebraic Reasoning. The Case os the Shanxi Province of China. Acta Sci. (Canoas), v. 22, n. 1, p. 38-60, 2020.

TRCVIVO.INNOVASCHOOLS. TRC, 2019. Unidades. Disponible en: https://trcvivo.innovaschools.edu.pe/contenido. Acceso en: 10 mar. 2021. 